\title{
Hermafroditismo Funcional de la Gónada de Fissurella crassa (Mollusca: Fissurellidae)
}

\author{
Functional Hermaphrodite Gonad in Fissurella crassa (Mollusca: Fissurellidae)
}

"Alberto Olivares Paz; ${ }^{* *}$ David Jofré Madariaga; ${ }^{* *}$ Carlos Alvarez Mazú \& ${ }^{* * *}$ Eduardo Bustos-Obregón

OLIVARES, P. A.; JOFRÉ, M. D.; ÁLVAREZ, M. C. \& BUSTOS-OBREGóN, E. Hermafroditismo funcional de la gónada de Fissurella crassa (Mollusca: Fissurellidae). Int. J. Morphol., 27(2):509-514, 2009.

RESUMEN: La costa del Pacífico sudoriental es el hábitat de las 13 especies de lapas descritas del subgénero Fissurella Brugière. En estas especies no existe dimorfismo sexual, los animales son dioicos, el sexo se reconoce explorando directa o indirectamente las gónadas y no tienen procesos de reversión sexual. La presencia de un organismo de Fissurella crassa con gónada formada por porciones de ovario y otras de testículo con capacidad para generar óvulos y espermatozoides, evidencia la potencialidad que los organismos de Fissurella poseen para desarrollar el hermafroditismo funcional. Sin embargo, el presente hallazgo no permite inferir si el agente desencadenante del desarrollo sincrónico funcional de la gónada hermafrodita es un factor endógeno y/o asociado a algún evento exógeno medio ambiental.

PALABRAS CLAVE: Mollusca; Fissurella crassa; Hermafrodita; Gónada; Gametos.

\section{INTRODUCCIÓN}

La costa litoral del Pacífico sudoriental es el hábitat para 13 especies del subgénero Fissurella Bruguièri; Fissurellidae:Vetigastropoda (McLean, 1984), de las cuales 10 son de importancia económica y forman parte de una pesquería multiespecífica artesanal de Chile (Bretos, 1988; Oliva \& Castilla, 1992; Osorio, 2002). Las diferentes especies de Fissurella son dioicas, carecen de dimorfismo sexual externo y el sexo solamente se reconoce después de la disección del animal, mediante la observación macroscópica del color de la gónada. El testículo presenta una amplia gama de coloración que fluctúa entre diferentes tonalidades de amarillo pálido a blanco lechoso y el ovario muestra variaciones en la intensidad del color verde (Bretos et al., 1988; Bretos, 1978; Bretos \& Chihuailaf, 1993). Un método alternativo para identificar el sexo, sin sacrificar al animal, consiste en la observación de células germinales obtenidas por medio de punciones gonádicas, a través del pie (Huaquín et al., 1998). En diferentes estudios realizados se ha constatado que las especies de Fissurella son dioicas y no presentan evidencias de inversión sexual ni de hermafroditismo (Bretos
\& Chihuailaf; Huaquín et al., 1998; Bretos et al., 1983). Sin embargo, en contraste a lo anterior en este trabajo se describe la organización de un organismo hermafrodita cuya gónada se caracteriza por tener una estructura y organización histológica de ovario y testículo, con capacidad funcional para generar óvulos y espermatozoides simultáneamente.

\section{MATERIAL Y MÉTODO}

Área de estudio. En la zona intermareal y submareal del litoral rocoso comprendida desde Isla Santa María (Lat. 232ㄱㅇ S; Long. $70^{\circ} 36^{\circ} \mathrm{W}$ ) hasta playa Escondida (Lat. $23^{\circ} 51^{`} \mathrm{~S}$; Long. $70^{\circ} 30^{` W}$ ), II Región de Antofagasta, Chile, se recolectaron individuos de diferentes tamaños de $F$. latimarginata, $F$. maxima, F. cumingi, F. costata, F. limbata y F. crassa (Figs. 1a-1b). Los ejemplares fueron clasificados en base a las características morfológicas y diseños de coloraciones específicas de la concha y del cuerpo descrita para las diferentes especies de Fissurella Bruguière (Osorio; Oliva \& Castilla, 1992).

\footnotetext{
* Departamento de Acuicultura. Facultad de Recursos del Mar, Universidad de Antofagasta, Casilla 170, Antofagasta, Chile.

** Instituto de Investigaciones Oceanológicas, Facultad de Recursos del Mar, Universidad de Antofagasta, Casilla 170, Antofagasta, Chile.

**** Laboratorio Biología de la Reproducción (ICBM), Facultad de Medicina, Universidad de Chile, Santiago Chile.

Financiado parcialmente por proyecto FONDECYT 1070898
} 
Morfometría y determinación del sexo. A cada uno de los ejemplares se les medió el largo de la concha (precisión 0,1 $\mathrm{mm}$ ). El sexo fue reconocido en animales disectados en concordancia a los patrones de coloración gonadal mencionados para machos y hembras (Bretos \& Chihuailaf). Se constató la proporción de los sexos considerando la relación 1:1 mediante la prueba de Chi-Cuadrado $\left(\mathrm{c}^{2}\right)$, con una significancia menor a 0.01 y un valor crítico de $\mathrm{c}^{2}$ igual a 6.635 (Zar, 1996).

Histología gonadal. A nivel de microscopía óptica se realizó el análisis histológico de secciones de gónada teñidas con hematoxilina-eosina. Las muestras fueron procesadas por técnica histológica de rutina, después de haber sido fijadas en Bouin alcohólico (Howard \& Smith, 1983).

\section{RESULTADOS Y DISCUSIÓN.}

Se analizaron 700 ejemplares entre las 6 especies de Fissurella. Los ejemplares de mayor tamaño correspondieron a F. cumingi, F. latimarginata y F. maxima, en concordancia al denominado grupo $F$. maxima (McLean) y las más pequeñas a $F$. limbata y $F$. crassa (Tabla I). Todos los ejemplares eran adultos y en esta categoría las proporciones de sexos observadas en estas especies no variaron significativamente $(\mathrm{p}<0,01)$ de la proporción sexual 1:1 (Tabla I), lo cual ratifica que en el subgénero Fissurella Bruguièri no hay procesos de reversión sexual durante el crecimiento y maduración sexual.

Aunque en F. crassa; denominada popularmente "lapa de sol", "lapa florío", o "lapa ocho" según la región de Chile (Bretos, 1988); también se detectó un porcentaje similar de hembras y machos, adicionalmente se constató la existencia de un animal hermafrodita, lo cual difiere de las características sexuales descritas para las distintas especies del género Fissurella de aguas tropicales y temperadas (Ward, 1966; Huaquín et al., 2004; Collado \& Brown, 2007).
El animal hermafrodita fue recolectado en el intermareal frente a la Universidad de Antofagasta (Lat. $23^{\circ} 41^{`} 60^{\prime \prime} \mathrm{S}$; Long. $70^{\circ} 25^{`} 1,20^{\prime} \mathrm{W}$ ). Tenía las siguientes características morfométricas: largo total de concha $36,73 \mathrm{~mm}$; ancho de concha $21,36 \mathrm{~mm}$; alto de concha 7,71 mm., peso total 7,6685 g, peso de la concha 2,2366, peso del cuerpo $5,723 \mathrm{~g}$, peso del pie $3,85 \mathrm{~g}$ y peso de la gónada $0,2911 \mathrm{~g}$. De acuerdo a la curva de crecimiento determinada para esta especie el animal hermafrodita tendría una edad aproximada de 21 meses (Bretos, 1980).

Fig. 1a. Zona del intermareal y submareal de un sector del litoral de la costa de Antofagasta, Chile, en la cual habitan distintas especies de Fissurella.

Fig. 1b. Características morfológicas de organismos correspondientes a tres especies de Fissurella de la costa de Antofagasta, Chile (barra representa $5 \mathrm{~cm}$ ).

Figs. 2-4. Aspecto macroscópico y patrón de coloración del testículo (Fig. 3), ovario (Fig. 3) y de la gónada hermafrodita (Fig. 4) de Fissurella (barra equivale a $1 \mathrm{~cm}$ ).

Figs. 5-8. Cortes de $6 \mu \mathrm{m}$ de gónadas de F. crassa, teñidos con Hematoxilina y Eosina. La barra corresponde a $100 \mu \mathrm{m}$ ). Las microfotografías fueron registradas en cámara digital.

Fig. 5. Sección de ovario con numerosos ovocitos maduros (Om) que llenan el acino ovárico y ovocitos no vitelados (Onv) en la parte interna de la pared de los ácinos.

Fig. 6. Sección de testículo maduro en fase de liberación moderada de espermatozoides (Es). El centro de los túbulos seminíferos ocupado por vaso sanguíneo (VS) alrededor del cual se organiza el estrato germinal (Eg).

Fig. 7. Sección de gónada del animal hermafrodita, a través de la porción del ovario y testículo. Los ovocitos maduros que ocupan completamente la cavidad del alveolo y los túbulos seminíferos (Ts) liberando espermatozoides evidencian la función gamética de ambas gónadas.

Fig. 8. Sección de túbulos seminíferos, de la gónada hermafrodita, en etapa inicial de liberación de espermatozoides.

Tabla I. Longitud promedio (X \pm D.E.) de la concha y valores de $\mathrm{c}^{2}$ para las proporciones sexuales de las diferentes especies de Fissurella, que habitan en el litoral de Antofagasta, Chile.

\begin{tabular}{|c|c|c|c|c|c|c|c|c|}
\hline \multirow[t]{2}{*}{ Especie } & \multicolumn{2}{|c|}{ Largo concha (mm) } & \multicolumn{2}{|c|}{ Machos (M) } & \multicolumn{2}{|c|}{ Hembras (H) } & \multicolumn{2}{|c|}{ Proporción Sexual } \\
\hline & $\mathrm{n}$ & $\mathbf{X} \pm$ D.E. & $\mathrm{n}$ & $\%$ & $\mathrm{n}$ & $\%$ & $\mathrm{M} / \mathrm{H}$ & $c^{2}$ \\
\hline F. cumingi & 57 & $71,6 \pm 8,9$ & 27 & 47,36 & 30 & 52,63 & 0,90 & 0,16 \\
\hline F. maxima & 188 & $68,7 \pm 14,7$ & 78 & 41,49 & 110 & 58,51 & 0,71 & 5,68 \\
\hline F. latimarginata & 141 & $67,9 \pm 9,4$ & 75 & 53,19 & 66 & 46,81 & 1,14 & 0,57 \\
\hline F. costata & 50 & $55,0 \pm 7,3$ & 30 & 60,00 & 20 & 40,00 & 1,50 & 2,00 \\
\hline F. limbata & 61 & $60,9 \pm 9,8$ & 30 & 49,18 & 31 & 50,82 & 0,97 & 0,02 \\
\hline F. crassa & 203 & $53,5 \pm 9,8$ & 93 & 45,81 & 110 & 54,19 & 0,85 & 1,42 \\
\hline
\end{tabular}


OLIVARES, P. A.; JOFRE, M. D.; ÁLVAREZ, M. C. \& BUSTOS-OBREGóN, E. Hermafroditismo funcional de la gónada de Fissurella crassa (Mollusca: Fissurellidae). Int. J. Morphol., 27(2):509-514, 2009.
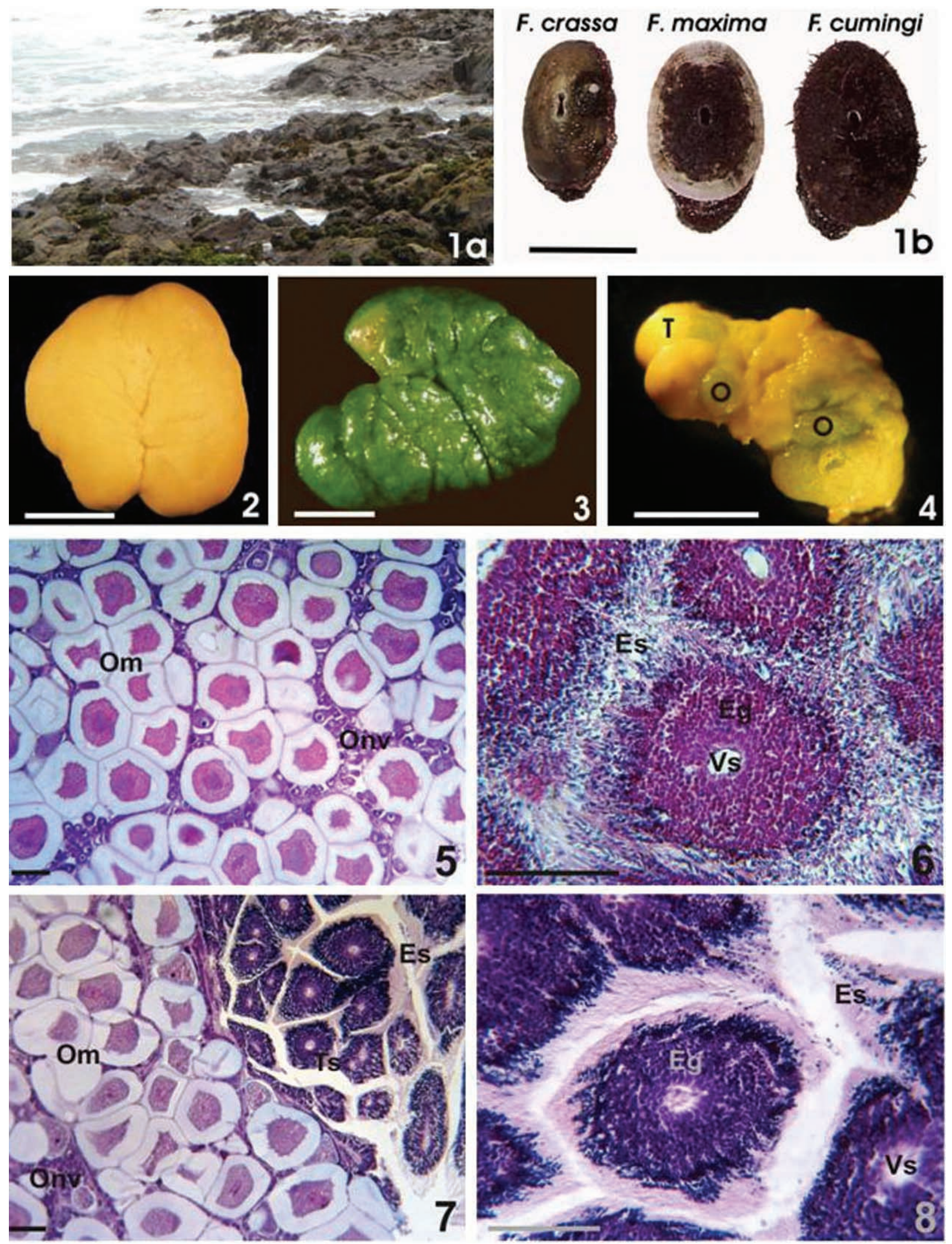
Análisis macroscópico de las gónadas: los machos de las distintas especies analizadas en este estudio presentaron un testículo, de coloración blanquecina a amarillo pálido, dividido superficialmente en lóbulos irregulares (Fig. 2). A su vez, las hembras tenían un solo ovario de color verde, cuya superficie está recorrida por surcos superficiales poco profundos que lo dividen parcial e irregularmente (Fig. 3). Estos caracteres macroscópicos del ovario y testículo se corresponden a los patrones característicos definidos para las gónadas de las diferentes especies del género Fissurella, en las que se ha analizado el ciclo reproductivo y funcionamiento gametogénico gonadal (Bretos \& Chihuailaf; Huaquín et al., 2004, Collado \& Brown). El animal hermafrodita presentó una gónada que difiere drásticamente de la forma acorazonada y sacciforme del ovario y testículo. Esta gónada atípica y de forma irregular se caracterizó por estar estructurada en base a numerosas protuberancias semicirculares de color blanquecino y otras de color verde, en concordancia con las variaciones de los colores del testículo y ovario respectivamente (Fig. 4).

Análisis microscópico: en todas las especies analizadas la gónada de ambos sexos está formada por una serie de septos de tejido conectivo en torno al cual se disponen las células germinales, en una organización característica descrita para las especies del género Fissurella (Oliva, 1992; Collado \& Brown). En el ovario los diversos septos presentan ovogonias, ovocitos no vitelados de diferentes tamaños, ovocitos vitelados y maduros (Fig. 5). En el testículo, las células germinales muestran una distribución centrifuga irradiando desde el centro hacia afuera en estratos secuenciales las espermatogonias, espermatocitos I, espermatocitos II, espermátidas redondas y espermátidas en diferenciación, las cuales se distribuyen en columnas perpendiculares y durante la espermiación se desorganizan desde el extremo apical al basal (Fig. 6). La gónada hermafrodita estaba estructurada en diferentes áreas separadas por una fina capa de tejido conectivo, correspondientes a organizaciones histológicas de ovario o de testículo (Fig. 7), de acuerdo con los respectivos patrones macroscópicos de color descritos para cada uno de los órganos. Las porciones ováricas poseen todos los tipos de células que mues- tran la progresión normal de la formación de ovocitos a partir de ovogonias. La presencia de ovocitos vitelados maduros que llenan completamente los acinos ováricos son indicadores de que el funcionamiento de las diferentes organizaciones ováricas de la gónada hermafrodita no difiere de la función gametogénica del ovario presente en organismos hembras. Similarmente, en las porciones de testículo del hermafrodita todos los túbulos seminíferos tienen las distintas poblaciones de células germinales que facultan la formación de espermatozoides a partir de las espermatogonias. El evento de espermiación de los túbulos seminíferos (Fig. 8) evidencia que el animal hermafrodita tendría la potencialidad de desovar y eventualmente reproducirse. Así se puede inferir que la gónada hermafrodita es gametogénicamente funcional, existiendo un desarrollo sincrónico en la formación de gametos maduros, aspecto que ha sido constatado en otros moluscos (Borzone et al., 2003). No obstante, no se puede aseverar si la liberación de óvulos y espermatozoides serían coincidentes o si paralelamente se hubiere desencadenado algún mecanismo que interfiera en la liberación simultánea de gametos, como la evacuación protándrica que minimiza las posibilidades de la autofecundación en moluscos hermafroditas (Avendaño et al., 2001; Villalejo \& Ochoa, 1993).

En adición, no es posible identificar el o los agentes internos y/o externos que hayan desencadenado el desarrollo de una gónada hermafrodita en este ejemplar de Fissurella crassa. Esta especie es un habitante característico de la zona media intermareal (Serra et al., 2001) las cuales en todo el mundo son susceptibles a los diversos agentes contaminantes derivados de la actividad humana y entre ellos algunos han sido identificados como desencadenantes de anormalidades en la estructura y funcionamiento reproductivo de moluscos (Thompson et al., 2002). El litoral de La bahía de San Jorge (Antofagasta, II Región), no difiere de esta situación y está sometida a los efectos de una moderada acción antropogénica; destacando los altos aportes naturales de metales pesados y los provenientes por descargas industriales y municipales, que tienden a ser acumulados en alta cantidad en los tejidos de algunos invertebrados que habitan la zona (Salamanca et al. 2004).

OLIVARES, P. A.; JOFRE, M. D.; ÁLVAREZ, M. C. \& BUSTOS-OBREGóN, E. Functional hermaphrodite gonad in Fissurella crassa (Mollusca:Fissurellidae). Int. J. Morphol., 27(2):509-514, 2009.

SUMMARY: The coast of the Southeastern Pacific is the habitat for 13 species of described keyhole limpets of the subgenus Fissurella Brugière. In these species sexual dimorphism does not exist, the animals are dioicos, the sex is recognized exploring directly or indirectly the gonads and they do not have processes of sexual reversion. The presence of an organism Fissurella crassa with portions of ovary and testicle with ability to generate ova and sperms, demonstrates the potential that Fissurella's organisms possess to develop functional hermaphroditism. Nevertheless, the present find does not allow to infer if the trigger agent of the synchronous functional development of the hermaphrodite gonad is a factor endogenous and / or associated with any exogenous environmental event.

KEY WORDS : Mollusca; Fissurella crassa; Hermaphrodite; Gonad; Gamete. 


\section{REFERENCIAS BIBLIOGRÁFICAS}

Avendaño, M.; Le Pennec, M. \& Cantillanez, M. Anormalidades en larvas de Argopecten purpuratus (Lamarck, 1819) (Mollusca:Pectinidae), uno de los problemas en la producción artificial de semilla. Estudios Oceanológicos, 20:33-42, 2001.

Borzone, C. A.; Pezzuto, P. R. \& Garcia Tavares, Y. A. Características histológicas del ciclo reproductivo de Euvola ziczac (Linnaeus) (Pectinidae, Bivlavia) del litoral sur-sudeste del Brasil. Revista Brasileira de Zoologia, 20(4):763-72, 2003.

Bretos, M.; Tesorieri, I. \& Alvarez, L. The biology of Fissurella maxima Sowerby (Mollusca: Archaeogastropoda) in northern Chile. 2. Notes ion its reproduction. Biology Bulletin, 165:559-68, 1983.

Bretos, M. Age determination in the keyhole limpet Fissurella crassa Lamarck (Archaeogastropoda: Fissurellidae), based on shell growth rings. Biology Bulletin, 159:606-12, 1980.

Bretos, M. \& Chihuailaf, R. Studies on the reproduction and gonadal parasites of Fissurella pulchra (Gastropoda: Prosobranchia). The Veliger, 36(3):24551, 1993.

Bretos, M. Growth in the keyhole limpet Fissurella crassa Lamarck (Mollusca : Archaeogastropoda). The Veliger, 21(2):268-73, 1978.

Bretos, M.; Gutiérrez, J. \& Espinoza, Z. Estudios biológicos para el manejo de Fissurella picta. Medio Ambiente, 9(1):28-34, 1988.

Bretos, M. Pesquería de lapas en Chile. Medio Ambiente, $9(2): 7-12,1988$.

Collado, G. A. \& Brown, D. I. Microscopic anatomy of the reproductive system in two sympatric species of Fissurella Brugièri, 1789 (Mollusca:Vetigastropoda). Int. J. Morphol., 25(2):315-22, 2007.

Howard, D. W. \& Smith, C. S. Histological techniques for marine bivalve molluscs. Massachusetts, NOAA Technical Memorandum, 1983.

Huaquín, L.; Guerra, R. \& Bretos, M. Identificación del sexo y morfología de gametos de la lapa Fissurella crassa Lamarck, 1822 (Mollusca: Archaeo- gastropoda). Rev. Biol. Mar. Oceanogr., 33(2):22339, 1988.

Huaquin, L.; Guerra, R. \& Del Campo, A. Ovary cell differentiation and morphology of mature oocytes in Fisurella crassa Lamarck 1822 (Mollusca: Archaeogastropoda). Invert. Rep. Dev., 46:103-10, 2004.

McLean, J. H. Systematics of Fissurella in the Peruviana in Magellanic faunal provinces (Gastropoda: Prosobranquia). Natural History Museum of Los Angeles County, Science Bulletin, 354:1-70, 1984.

Oliva, D. \& Castilla, J. C. Guía para el reconocimiento y morfometría de diez especies del género Fissurella Bruguiere, 1789 (Mollusca:Gastropoda) comunes en la pesquería y conchales indígenas de Chile central y sur. Gayana Zoo., 56(3-4):77-108, 1992.

Oliva, M. Parasitic castration in Fissurella crassa (Archaeogastropoda) due to an adult Digenea Proctoeces lintoni (Fellodistomidae)). Memóries do Instituto Oswaldo Cruz, 87(1):37-42, 1992.

Osorio, R. C. Moluscos marinos en Chile. Especies de importancia económica. Guía para su identificación. Ed. Facultad de Ciencias, Universidad de Chile, Santiago Chile, 2002.

Salamanca, M. A.; Jara, B. \& Rodriguez, T. Niveles de $\mathrm{Cu}, \mathrm{Pb}, \mathrm{y} \mathrm{Zn}$ en agua y Perumytilus purpuratus en Bahía San Jorge, Norte de Chile. Gayana, 68(1):5362, 2004.

Serra, G.; Chelazzi, G. \& Castilla, J. C. Temporal and spatial activity of the key-hole limpet Fissurella crassa (Mollusca: Gastropoda) in the eastern Pacific. J. Mar. Biol. Ass. U.K., 81:485-90, 2001.

Thompson, R. C.; Crowe, T. P. \& Hawkins, S. J. Rocky intertidal comumunities: past environmental changes, present status and predictions for the next 25 years. Enviromental Conservation, 29(2):168-91, 2002.

Villalejo-Fuerte, M. \& Ochoa-Báez, R. I. El ciclo reproductivo de la almeja catarina, Argopecten circularis (Sowerby, 1835), en relación con temperatura y fotoperiodo, en Bahía Concepcion, B.C.S., México. Ciencias Marinas, 19(2):181-02, 1993. 
OLIVARES, P. A.; JOFRE, M. D.; ÁLVAREZ, M. C. \& BUSTOS-OBREGÓN, E. Hermafroditismo funcional de la gónada de Fissurella crassa (Mollusca: Fissurellidae). Int. J. Morphol., 27(2):509-514, 2009

Ward, J. The breeding cycle of the keyhole limpet Fissurella barbadensis Gmelin. Bull. Mar. Sci., 16:685-95, 1966.

Zar, J. H. Biostatistical analysis. $3^{\text {a }}$. Ed. New Jersey, Prentice Hall, 1996.
Dirección para correspondencia:

Alberto Olivares Paz

Departamento de Acuicultura.

Facultad de Recursos del Mar

Universidad de Antofagasta

Casilla 170,

Antofagasta -CHILE

Email: aolivares@uantof.cl

Recibido : 02-01-2009

Aceptado: 12-03-2009 